\title{
Task switching is not cue switching
}

\author{
ERIK M. ALTMANN \\ Michigan State University, East Lansing, Michigan
}

\begin{abstract}
With the aim of reducing cognitive control in task switching to simpler processes, researchers have proposed in a series of recent studies that there is little more to switching tasks than switching cues. The present study addresses three questions concerning this reduction hypothesis. First, does switching cues account for all relevant variance associated with switching tasks? Second, how well does this hypothesis generalize beyond the experimental procedure from which it was developed? Third, how well does this new procedure preserve relevant measures such as task-switch cost? The answers (no; not very; not very) suggest that task switching does not reduce to cue switching.
\end{abstract}

Task-switching procedures are useful for studying cognitive control in situations in which a stimulus can be processed in different ways and the cognitive system has to organize itself in response to changing instructions about which way is currently correct. One common task-switching procedure is explicit cuing, in which the experimental participant performs a sequence of trials, each accompanied by a task cue indicating how to process the stimulus on that trial. For example, in the set of materials used here, the stimulus is a rectangle that can be judged either according to its height or (horizontal) width. The task cue presented on each trial (indicating height or width) is randomized in some way, setting up a comparison between no-switch trials, on which the task repeats from the previous trial, and task-switch trials, on which the task differs from the previous trial. If cognitive control processes are necessary to "reconfigure" (Monsell, 2003) or "program" (Logan, 2003) the system to perform one task as opposed to another, the difference in performance between no-switch and task-switch trials might seem to offer an index of such control processes in action (Monsell \& Driver, 2000).

A variant of explicit cuing that has been developed recently is the cue-switching procedure, in which cues are mapped 2:1 to tasks. For example, the letter $\mathrm{H}$ may be used to cue the height task on one trial, and the letter $U$ (for up-down) may be used to cue the same task on a different trial. (Analogous cues for the width task might be $\mathrm{W}$, for width, and $\mathrm{L}$, for left-right.) A 2:1 cue-task mapping allows comparisons between no-switch trials, taskswitch trials, and now cue-switch trials, on which the cue changes but the task does not. One interesting hypothesis that can be explored with this procedure is that task switching reduces to cue switching, in that, to perform

This work was supported by ONR Grant N00014-06-1-0077. The author thanks Rich Carlson and two anonymous reviewers for their rigorous comments and suggestions for improvement. Correspondence concerning this article should be addressed to E. M. Altmann, Department of Psychology, Michigan State University, East Lansing, MI 48824 (e-mail: ema@msu.edu). correctly on a given trial, the system needs only to encode the current task cue, encode the current trial stimulus, and then use the two together as a compound cue to retrieve a response from memory. This simple and elegant model receives some support from the fact that task-switch cost (the performance difference between no-switch and taskswitch trials) is often not much larger than cue-switch cost (the performance difference between no-switch and cueswitch trials; Logan \& Bundesen, 2003, 2004; Schneider \& Logan, 2005).

This report addresses three questions concerning the cue-switching procedure and the associated compoundcue model. The first question is largely empirical: Does switching tasks cause behavioral variance that is not caused by switching cues? This question is addressed here by modifying the usual trial line of explicit-cuingtype experimental procedures. Here, trials are organized into pairs, with a Trial 1 followed by a Trial 2 . The task cue onsets prior to Trial 1 and offsets with the Trial 1 response, at which point the Trial 2 stimulus onsets immediately and the participant reapplies the cue from Trial 1. Cue-switch cost on Trial 2 of this design would not be expected, because cue encoding should be complete by this point. Under the compound-cue model, the cue must have been encoded to retrieve the Trial 1 response, and is, in any case, no longer perceptually available. Of interest is whether Trial 2 shows task-switch cost. In previous studies, an error task-switch cost has been found on Trials 2 and later (Altmann, 2002, 2004a), but no evidence has yet been developed on a corresponding effect in terms of latencies, which have been the measure of primary interest in cue-switching studies. If latency task-switch cost is evident on Trial 2 but cue-switch cost is not, then cue switching would seem to be incomplete as an account of task switching.

The second question is primarily theoretical: Does the formal specification of the compound-cue model generalize to performance with the more common 1:1 cue-task mapping? There is evidence from existing 1:1 studies that at least one quantitative prediction of the model is incorrect. The model predicts that the trial-to-trial transition 
variable, with levels task-switch and no-switch, should interact pervasively with another variable commonly manipulated in this domain, the cue-stimulus interval (CSI) separating cue onset from trial stimulus onset (Logan \& Bundesen, 2003, 2004; Schneider \& Logan, 2005). This CSI $\times$ transition interaction has been characterized as pervasive (Logan, 2003; Monsell, 2003), but is often not reliable (Altmann, 2004a, 2004b; Koch, 2001; Meiran \& Marciano, 2002; Poljac, de Haan, \& van Galen, 2006), which is a problem for the compound-cue model that has not yet been addressed. Fortunately, the model makes quantitative predictions for this interaction that can be tested against empirical data using inferential statistics.

A third, largely methodological question relates to the cue-switching procedure as an instance or representative of task-switching procedures more broadly: Do the usual behavioral measures of interest behave the same way with a 2:1 cue-task mapping as with the more common $1: 1$ mapping? Suppose, for example, that task-switch cost changes when redundant cues are added to make a 2:1 mapping. This would seem to require that a model developed to account for 2:1 task-switch cost would have to be elaborated in some way to generalize to $1: 1$ task-switch cost-especially if the model's account of task-switch cost happens to rest heavily on assumptions about how cues are processed.

The experiment reported below compares two betweenparticipants conditions: one in which cues are mapped 2:1 to tasks (representing the cue-switching procedure) and one in which cues are mapped 1:1 to tasks (representing the explicit-cuing procedure). CSI is manipulated within participants, to test predictions of the compoundcue model concerning the CSI $\times$ transition interaction. The resulting data address the first and third questions raised above, concerning Trial 2 performance and mapping effects on (Trial 1) task-switch cost, respectively. The compound-cue model is then fit to the data to address the second question, concerning the ability of the model to generalize beyond the cue-switching procedure.

\section{METHOD}

\section{Participants}

Thirty Michigan State University undergraduates participated, with 15 randomly assigned to each cue-task mapping condition. Four additional participants were replaced because their sessionwise accuracy was below $90 \%$.

\section{Materials}

A task cue was the capital letter $\mathrm{H}, \mathrm{U}, \mathrm{W}$, or $\mathrm{L}$, displayed in a sans serif font with a height of $1 \mathrm{~cm}$. A trial stimulus was one of four rectangles formed by crossing dimensions $2.5 \mathrm{~cm}$ and $5.0 \mathrm{~cm}$. The cues were drawn in white and the stimuli in gray; both were on a dark background and centered on the display (with the stimulus surrounding the cue).

$\mathrm{H}$ and $\mathrm{U}$ cued the participant to judge the height (up and down) of a rectangle as tall or short, relative to the other rectangles in the set. $W$ and $L$ cued the participant to judge the width (left to right) of a rectangle as thick or thin. $\mathrm{H}$ and $\mathrm{W}$ were used in the 1:1 mapping condition. The " $Z$ " and " $p$ " keys of a standard keyboard were used for responding. The response-to-key mapping was randomized between participants.

\section{Procedure}

Each session began with an online introduction to the task environment, followed by a practice block with the experimenter present to answer any questions. The participants were told the total number of blocks, and were advised to take breaks between blocks if necessary.

Trials were grouped into pairs of two, with each pair preceded by a cue indicating the task for that pair. The task was randomly selected for every pair, and in the 2:1 condition, a cue was randomly selected after the task was selected. A cue was visible for a CSI of either $100 \mathrm{msec}$ or $900 \mathrm{msec}$; the interval was also randomly selected for every pair, prior to onset of the Trial 1 stimulus. The cue remained visible until the Trial 1 response, at which point the cue and stimulus offset together and the Trial 2 stimulus onset immediately. The Trial 2 response triggered a blank screen for a brief interval and then the next task cue. The blank screen interval and the CSI always summed to $1 \mathrm{sec}$.

There were 36 trial pairs to a block and 24 nonpractice blocks to a session. The participants received feedback only after blocks. If they scored below $90 \%$ on a block, they were asked to be more accurate, and if they scored $100 \%$, they were asked to see if they could go faster.

\section{Design and Analysis}

The main ANOVA included factors mapping (1:1, 2:1), CSI (short, long), and transition (no-switch, task-switch). (A task factor, with levels height and width, was also included, but is not reported here.) Mapping was between participants, and all other variables were random within blocks. This design was applied separately to Trials 1 and 2 and to latencies and errors. The practice block and the first trial of each remaining block were excluded from analysis. Latency data for Trial 1 were means of trials with correct responses; for Trial 2, they were means of trials with correct responses following correct responses to Trial 1 . Additional contrasts were used to probe specific questions, as described below.

\section{RESULTS}

Mean response latencies for each condition appear in Figure 1.

On Trial 1, CSI and transition effects were both larger in the $2: 1$ condition than in the $1: 1$ condition. There was a CSI $\times$ mapping interaction $\left[F(1,28)=17.7, M S_{\mathrm{e}}=\right.$ $23,131, p=.000]$, with a smaller CSI effect in the $1: 1$ condition (202 msec) than in the 2:1 condition $(361 \mathrm{msec}$ ), and a transition $\times$ mapping interaction $[F(1,28)=6.3$, $\left.M S_{\mathrm{e}}=68,759, p=.018\right]$, with task-switch cost smaller in the $1: 1$ condition $(194 \mathrm{msec})$ than in the $2: 1$ condition $(360 \mathrm{msec})$. The CSI $\times$ transition interaction was marginal $\left[F(1,28)=3.9, M S_{\mathrm{e}}=19,933, p=.059\right]$, with switch cost slightly larger at short CSIs $(310 \mathrm{msec})$ than at long CSIs $(245 \mathrm{msec})$. There were main effects of CSI $\left[F(1,28)=210.8, M S_{\mathrm{e}}=23,131, p=.000\right]$, with short CSI $(1,235 \mathrm{msec})$ slower than long CSI $(953 \mathrm{msec})$, and transition $\left[F(1,28)=67.8, M S_{\mathrm{e}}=68,759, p=\right.$ $.000]$, with task-switch $(1,233 \mathrm{msec})$ slower than noswitch $(956 \mathrm{msec})$. There was no main effect of mapping $\left[F(1,28)=2.8, M S_{\mathrm{e}}=684,248, p=.103\right]$.

As a check on the possible influence of cues unique to the 2:1 condition, the ANOVA above was repeated with $\mathrm{U}$ and $\mathrm{L}$ trials excluded, so that all comparisons were between $\mathrm{H}$ and $\mathrm{W}$ trials. Only one outcome changed: The marginal CSI $\times$ transition interaction became nonsignifi$\operatorname{cant}\left[F(1,28)=2.0, M S_{\mathrm{e}}=30,603, p=.172\right]$. 


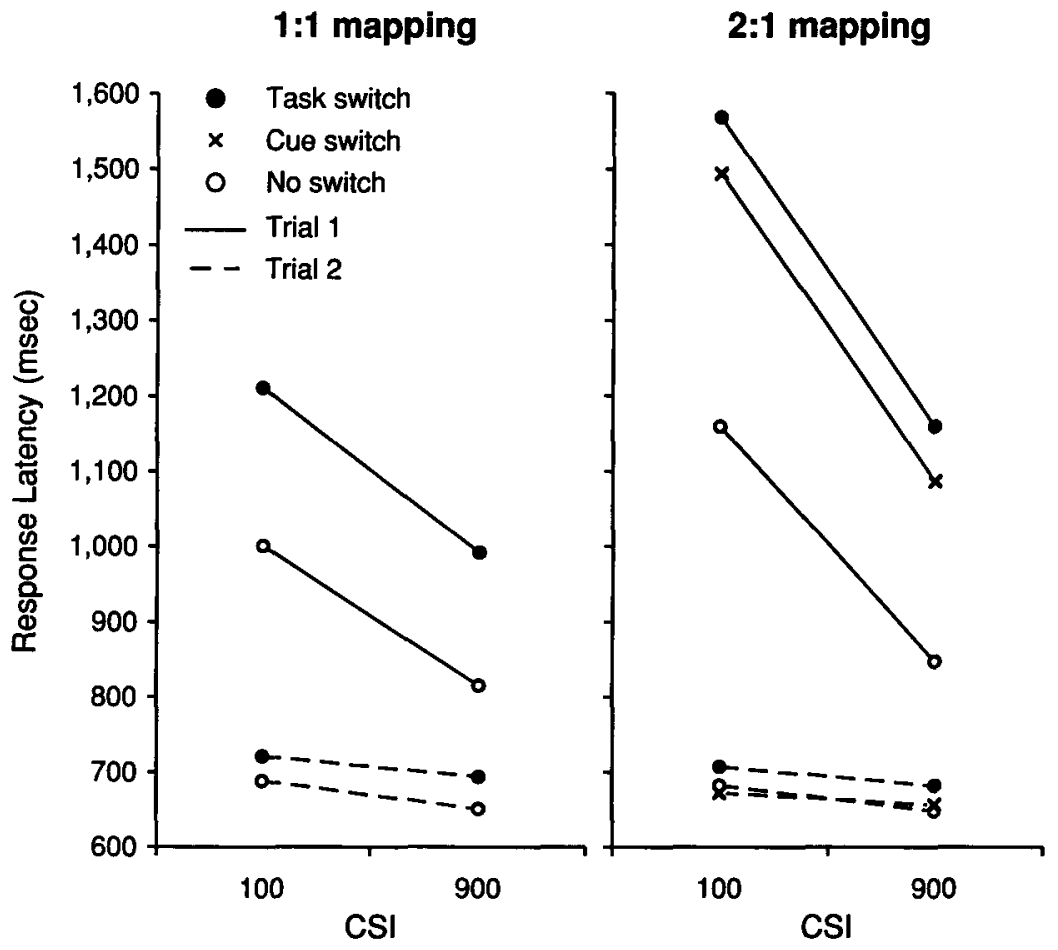

Figure 1. Mean response latencies from the experiment. CSI, cue-stimulus interval; 1:1 mapping, 1 cue per task; 2:1 mapping, 2 cues per task; Trial 1, first trial of a sametask pair; Trial 2, second trial of a same-task pair.

For use in the model-fitting exercise described later, separate ANOVAs were conducted within each mapping condition. The relevant finding was that the CSI $\times$ transition interaction was not reliable in either case. In the 1:1 condition, task-switch cost was not reliably different at short CSIs $(210 \mathrm{msec})$ than at long CSIs $(177 \mathrm{msec}$; $\left.F<1, M S_{\mathrm{e}}=9,393\right)$. Likewise, in the $2: 1$ condition, task-switch cost was not reliably different at short CSIs $(409 \mathrm{msec})$ than at long CSIs $(312 \mathrm{msec})[F(1,14)=3.0$, $\left.M S_{\mathrm{e}}=30,474, p=.104\right]$. In the task-switching literature, this particular interaction has been characterized as a "basic phenomenon" (Monsell, 2003) or "standard effect" (Logan \& Bundesen, 2003, 2004), but these and other results suggest that it is actually fickle, a point addressed again in the Discussion.

For comparison with previous cue-switching studies, cue-switch trials were compared with other transition types in the 2:1 condition. Cue-switch trials $(1,290 \mathrm{msec})$ were slower than no-switch trials $(1,003 \mathrm{msec})[F(1,14)=$ 45.7, $\left.M S_{\mathrm{e}}=53,956, p=.000\right]$ and marginally faster than task-switch trials $(1,364 \mathrm{msec})\left[F(1,14)=4.1, M S_{\mathrm{e}}=\right.$ $43,288, p=.063$ ], basically replicating previous results (e.g., Arrington \& Logan, 2004; Logan \& Bundesen, 2004; Mayr \& Kliegl, 2003).

On Trial 2, there was task-switch cost but no cue-switch cost, indicating a residual effect of switching tasks that is not explained by switching cues. Task-switch trials $(701 \mathrm{msec})$ were slower than no-switch trials $(667 \mathrm{msec})[F(1,14)=$ $12.4, M S_{\mathrm{e}}=7,541, p=.002$ ], but within the $2: 1$ condition, cue-switch trials (664 msec) were no different from noswitch trials ( $665 \mathrm{msec})\left(F=0.00, M S_{e}=5,967\right)$ and were faster than task-switch trials $(694 \mathrm{msec})[F(1,14)=7.6$, $\left.M S_{\mathrm{e}}=7,001, p=.016\right]$. There was also a CSI effect, with short CSIs $(700 \mathrm{msec})$ slower than long CSIs $(669 \mathrm{msec})$ $\left[F(1,28)=12.4, M S_{\mathrm{e}}=4,155, p=.002\right]$.

The error data, shown in Figure 2, do not contradict the latency results. On Trial 1, there was only a main effect of transition, with the error rate higher on task-switch trials $(2.31 \%)$ than on no-switch trials $(1.41 \%)[F(1,28)=$ $\left.17.8, M S_{e}=2.69, p=.000\right]$. Within the $2: 1$ condition, cue-switch errors (1.22\%) did not differ from no-switch errors $(1.51 \%)\left(M S_{\mathrm{e}}=1.96, p=.277\right)$, but were less frequent than task-switch errors $(2.61 \%)[F(1,14)=10.0$, $\left.M S_{\mathrm{e}}=5.81, p=.007\right]$. On Trial 2, no contrasts were reliable $(p>.15$ for all). Finally, the effect of trial was examined here; Trial 1 errors $(1.86 \%)$ were less frequent than Trial 2 errors $(3.32 \%)\left[F(1,28)=16.8, M S_{\mathrm{e}}=15.29\right.$, $p=.000]$.

\section{Model Fitting}

The formal representation of the compound-cue model was fit to these results. The processing assumptions of this model are that task cue onset triggers a cue-encoding process with exponentially distributed finishing times, which is followed by trial processing, during which the trial stimulus is encoded and the cue and stimulus representations are used together as a compound cue to retrieve a response. Under these assumptions, cue encoding can 


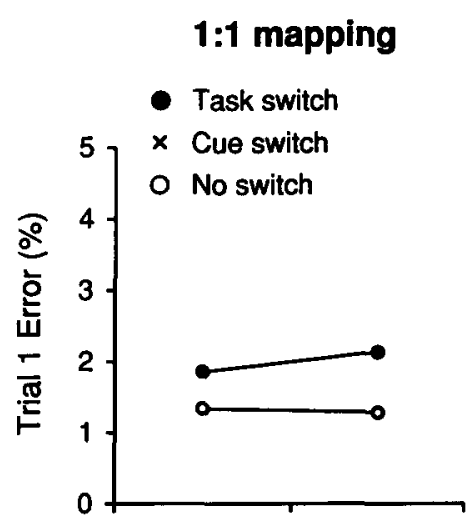

\section{2:1 mapping}
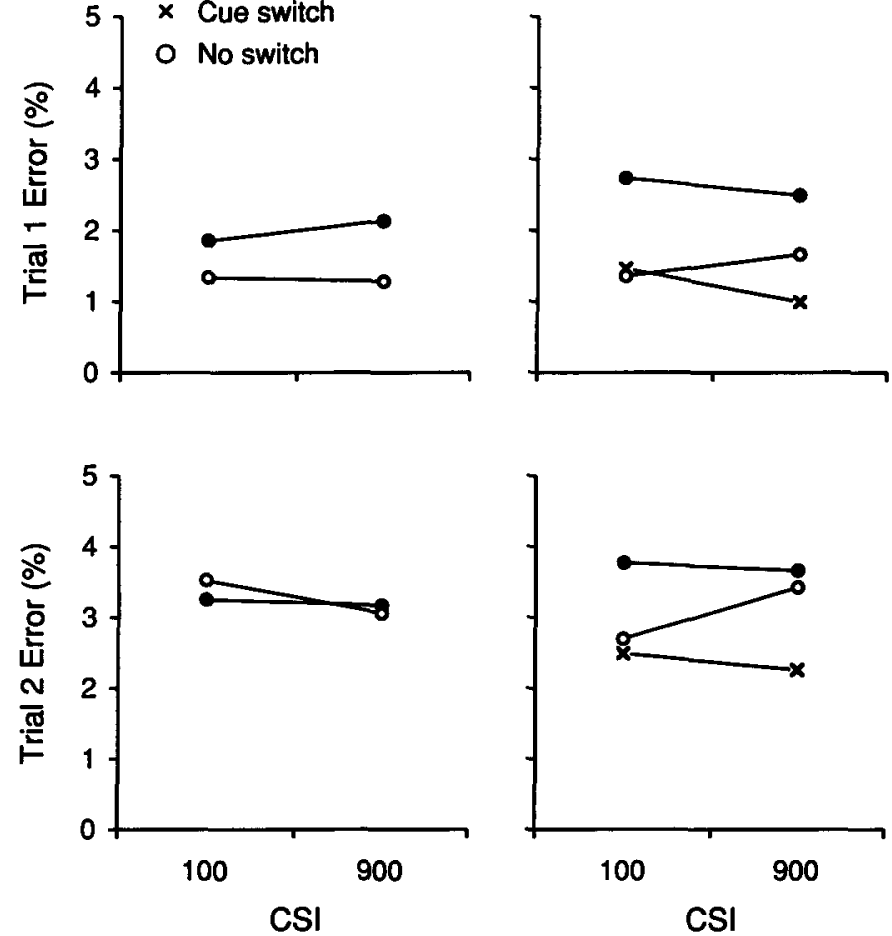

Figure 2. Error percentages from the experiment. CSI, cue-stimulus interval; 1:1 mapping, 1 cue per task; 2:1 mapping, 2 cues per task; Trial 1, first trial of a same-task pair; Trial 2, second trial of a same-task pair.

finish during the CSI, leaving slack time before trial processing begins, or it can continue up to or past trial stimulus onset. This analysis predicts a mean Trial 1 response time (RT) of

$$
\mathrm{RT}=\mathrm{RTbase}+\mu \exp (-\mathrm{CSU} / \mu),
$$

where $\mu$ represents mean time for cue encoding to finish and RTbase represents the duration of trial processing (Logan \& Bundesen, 2003). To explain switch cost, the model assumes that cue encoding is faster when it is primed by repetition of the cue from the previous trial.

The model was fit to mean latencies for each participant. There were four empirical values (CSI crossed with transition) per participant per task (height, width), from which three model parameters were estimated: $\mu_{-} \mathrm{n}$, the mean cue-encoding time on no-switch trials; $\mu_{-} \mathrm{t}$, the mean cue-encoding time on task-switch trials; and RTbase, the time needed for trial processing. All parameters were allowed to vary freely to obtain the optimal fit, which was measured by minimizing the root mean squared deviation between empirical and modeled latencies. The high ratio of parameters to data points $(3: 4)$ allowed the model high flexibility to fit the data.

Figure 3 shows the fits. In the 1:1 condition, values of the model parameters, averaged over task and participant, were $\mu_{-} \mathrm{n}=242 \mathrm{msec}, \mu_{-} \mathrm{t}=494 \mathrm{msec}$, and RTbase $=$ $833 \mathrm{msec}$. In the $2: 1$ condition, the values were $\mu \_\mathrm{n}=$ $546 \mathrm{msec}, \mu_{-} \mathrm{t}=1,005 \mathrm{msec}$, and RTbase $=684 \mathrm{msec}$. Participant-level data and parameters are available at www.msu.edu/ ema/cueswitching/.

The model fit the 2:1 condition well, but not the 1:1 condition. An ANOVA with source (modeled, empirical) as a within-participants variable revealed a source $\times$ mapping $\times$ transition interaction $\left[F(1,28)=9.1, M S_{\mathrm{e}}=639\right.$, $p=.005]$, meaning that the model fit task-switch costs differently in the two mapping conditions. In the 1:1 condition, there was an effect of source $[F(1,14)=167.6, p=$ $.000]$ and there were interactions of source and transition $\left[F(1,14)=14.2, M S_{\mathrm{e}}=993, p=.002\right]$, source and CSI $\left[F(1,14)=6.5, M S_{\mathrm{e}}=1,180, p=.023\right]$, and source, CSI, and transition $\left[F(1,14)=11.3, M S_{\mathrm{e}}=4,765, p=.005\right]$. In the 2:1 condition, there was no effect of source $(F<1)$ and no reliable interactions involving source.

\section{DISCUSSION}

The first question raised in the introduction was whether task switching produced behavioral variance that is not explained by cue switching, specifically on the uncued Trial 2 of a pair. Trial 2 latencies did in fact show a robust task- 

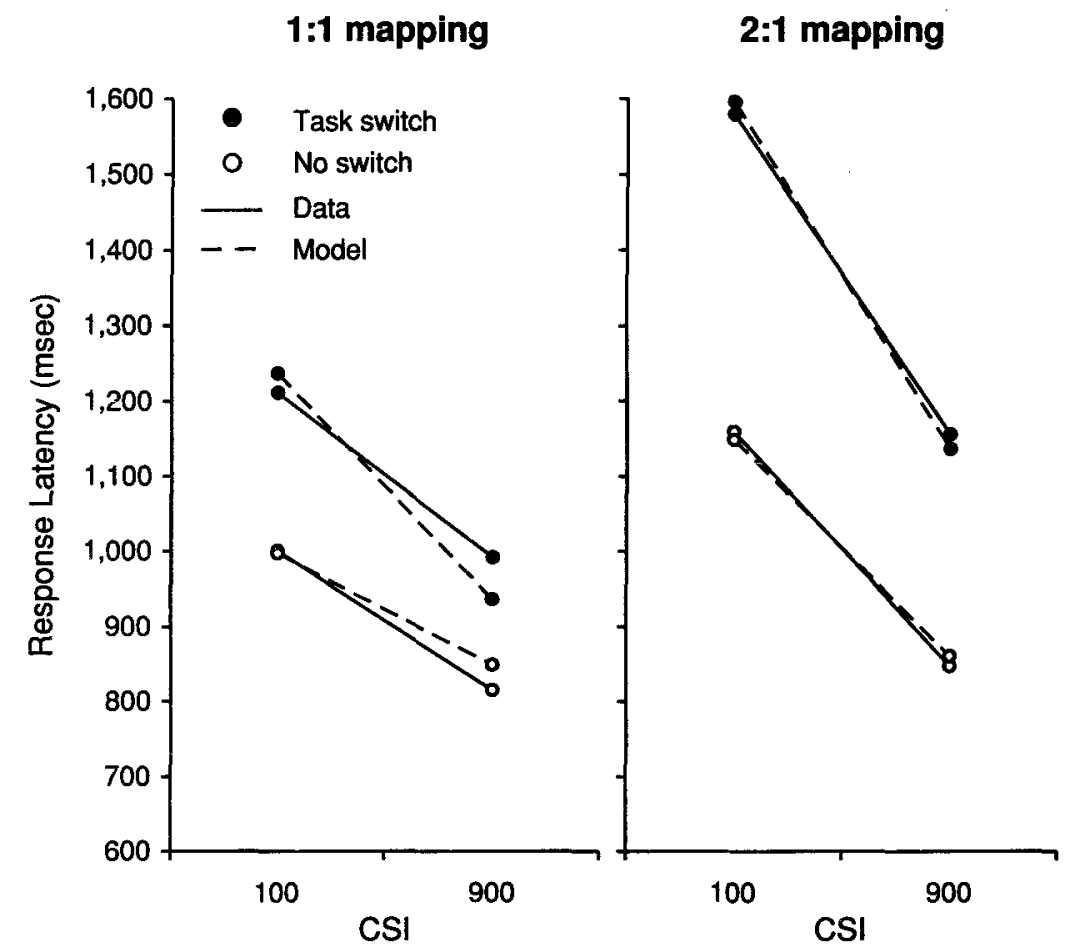

Figure 3. Fits of the compound-cue model to Trial 1 response latencies from Figure 1 (see text for fitting procedure). CSI, cue-stimulus interval; 1:1 mapping, 1 cue per task; 2:1 mapping, 2 cues per task.

switch cost (Figure 1, dashed lines), but zero cue-switch cost, consistent with an obvious account of processing in which the cue has been fully encoded by the end of Trial 1 . The indication is that more processes are involved in task switching than in cue switching. It is worth noting that a similar dissociation has been found in terms of the reverse response repetition effect, in which the usual facilitation found with response repetition is attenuated or even reversed by a task switch (Kleinsorge, 1999; Mayr, 2002; Mayr \& Kliegl, 2003). Response repetition inhibited performance on task-switch trials and facilitated performance on cue-switch and no-switch trials alike (Mayr \& Kliegl, 2003), again indicating an effect caused by switching tasks that is not caused by switching cues. These results, together with the fact that task-switch cost is often significantly greater than cue-switch cost (Arrington \& Logan, 2004; Logan \& Bundesen, 2004; Mayr \& Kliegl, 2003), suggest that cue switching accounts for, at best, a subset of the variance associated with task switching (and, at worst, a different set).

The second question raised was how well the formal compound-cue model was able to fit data from a 1:1 cue-task mapping, which remains the modal procedural variant used in task-switching studies. The model overpredicted the CSI $\times$ transition interaction in the $1: 1$ condition (Figure 3, left panel). To elaborate the theoretical picture somewhat, Figure 4 plots time-course functions that interpolate the model fits to a range of CSIs, for comparison against the model's RTbase estimates (dashed lines).
The model's CSI $\times$ transition interaction is sharper in the $1: 1$ condition (left panel) than in the $2: 1$ condition (right panel), because in the $1: 1$ condition, the no-switch timecourse function asymptotes at RTbase. This drop to asymptote reflects the combined influence of a low estimate for no-switch cue-encoding time, which corresponds to a low intercept for the no-switch time-course function, and a high estimate for RTbase for this mapping condition. In particular, this high estimate for RTbase, $833 \mathrm{msec}$, is substantially higher than the Trial 2 response latency, which was $677 \mathrm{msec}$ in this mapping condition. This discrepancy suggests that the RTbase estimate is inaccurate, given that Trial 2 latency spans at least stimulus encoding and response retrieval and thus represents an upper bound on the duration of trial processing. This independent check on RTbase, made possible by including an uncued Trial 2 in the present design, suggests that the compound-cue model does not always produce coherent estimates of basic processing parameters.

The deeper conceptual problem with the compoundcue model may be the basic assumption that predicts the CSI $\times$ transition interaction, which is that encoding can finish early (during the CSI) and thus leave slack time prior to trial stimulus onset. The resulting interaction should indeed be pervasive, because whenever CSI is manipulated, a longer CSI should always increase the relative frequency with which encoding finishes early, and thus should always modulate switch cost, given sufficient statistical power. However, here, as in other studies (Alt- 
1:1 mapping

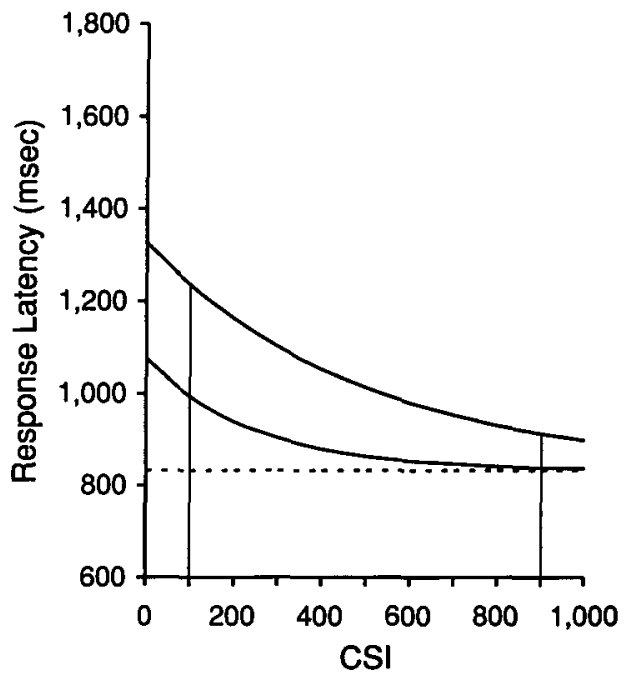

2:1 mapping

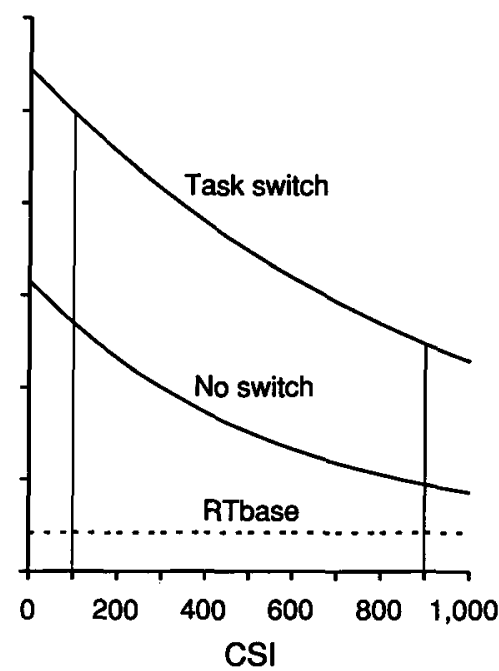

Figure 4. Time-course functions interpolated from the fits of the compound-cue model in Figure 3, and the model's estimates of RTbase (time needed for stimulus encoding and response retrieval). CSI, cue-stimulus interval; 1:1 mapping, 1 cue per task; 2:1 mapping, 2 cues per task.

mann, 2004a, 2004b; Koch, 2001; Meiran \& Marciano, 2002; Poljac et al., 2006), the interaction was not reliable, and in studies focusing specifically on this issue, power analyses have suggested that these null interactions were not Type II errors (Altmann, 2004a, 2004b). Conversely, the interaction was reliable in at least two of the three 1:1 studies to which the compound-cue model has been fit with some success ${ }^{1}$ (Experiments 2 and 5 of Logan \& Bundesen, 2003; no statistics seem to be reported for their Experiment 1). The apparent fickleness of this interaction is its own puzzle, and may be worth investigating in more detail to identify the full set of procedural variables that make it come and go and to identify the cognitive processes or parameters that are affected. For present purposes, the critical point is that the compound-cue model predicts that the CSI $\times$ transition interaction should be pervasive, whereas the evidence increasingly is that it is not.

The third question raised in the introduction concerns how well performance on the cue-switching procedure mirrors performance on the more common explicit-cuing procedure, with its simpler 1:1 mapping. Manipulating cues and tasks separately is supposed to "fractionate" task-switch cost into smaller components (see, e.g., Schneider \& Logan, 2005), but in fact it seems to make taskswitch cost larger, at least with the present set of materials. This increase in task-switch cost might reflect effects of cue-task mapping on the probability of actually switching tasks, which does influence task-switch cost (Mayr, 2006; Monsell \& Mizon, 2006; see also Dreisbach, Haider, \& Kluwe, 2002), but might also reflect the need for a new layer of processing or representation to cope with the more complex cue-task mapping. If the latter is the case, then when a manipulation in a cue-switching study shows some empirical outcome, what processes are being affected: the ones responsible for 1:1 task-switch cost, or a new set required specifically to cope with a 2:1 mapping? Some evidence on this question would be useful to develop before we try to use cue-switching data as a constraint on more general models of task switching.

In conclusion, there is reasonably diverse evidence, from this study alone, against the hypothesis that task switching reduces to cue switching. First, task switching produces behavioral variance in Trial 2 performance that is not explained by cue switching. Second, a formal model based on this reduction hypothesis predicts a CSI $X$ switching interaction that should be pervasive but in fact seems to come and go. Third, testing this hypothesis by manipulating cues as well as tasks seems to increase the effect of manipulating tasks, such that even a successful model of cue switching may explain something that is not actually task switching in a more usual sense. In the end, then, simple and elegant as it is, the reduction hypothesis may also just be wrong.

\section{REFERENCES}

Altmann, E. M. (2002). Functional decay of memory for tasks. Psychological Research, 66, 287-297.

AltmanN, E. M. (2004a). Advance preparation in task switching: What work is being done? Psychological Science, 15, 616-622.

AltmanN, E. M. (2004b). The preparation effect in task switching: Carryover of SOA. Memory \& Cognition, 32, 153-163.

Arrington, C. M., \& LogAN, G. D. (2004). Episodic and semantic components of the compound-stimulus strategy in the explicit taskcuing procedure. Memory \& Cognition, 32, 965-978.

Dreisbach, G., HaIder, H., \& KLUWE, R. H. (2002). Preparatory processes in the task-switching paradigm: Evidence from the use of prob- 
ability cues. Journal of Experimental Psychology: Learning, Memory, \& Cognition, 28, 468-483.

KLEINSORGE, T. (1999). Response repetition benefits and costs. Acta Psychologica, 103, 295-310.

КосH, I. (2001). Automatic and intentional activation of task sets. Journal of Experimental Psychology: Learning, Memory, \& Cognition, 27, 1474-1486.

LOGAN, G. D. (2003). Executive control of thought and action: In search of the wild homunculus. Current Directions in Psychological Science, $12,45-48$.

Logan, G. D., \& Bundesen, C. (2003). Clever homunculus: Is there an endogenous act of control in the explicit task-cuing procedure? Journal of Experimental Psychology: Human Perception \& Performance, 29, 575-599.

LoGAN, G. D., \& BundesEN, C. (2004). Very clever homunculus: Compound stimulus strategies for the explicit task-cuing procedure. Psychonomic Bulletin \& Review, 11, 832-840.

MAYr, U. (2002). Inhibition of action rules. Psychonomic Bulletin \& Review, 9, 93-99.

MAYR, U. (2006). What matters in the cued task-switching paradigm: Tasks or cues? Psychonomic Bulletin \& Review, 13, 794-799.

MAYR, U., \& KLIEGL, R. (2003). Differential effects of cue changes and task changes on task-set selection costs. Journal of Experimental Psychology: Learning, Memory, \& Cognition, 29, 362-372.

MeIran, N., \& Marciano, H. (2002). Limitations in advance task preparation: Switching the relevant stimulus dimension in speeded same-different comparisons. Memory \& Cognition, 30, 540-550.
Monsell, S. (2003). Task switching. Trends in Cognitive Sciences, 7, 134-140.

MONSELL, S., \& DRIVER, J. (2000). Banishing the control homunculus. In S. Monsell \& J. Driver (Eds.), Control of cognitive processes: Attention and performance XVIII (pp. 3-32). Cambridge, MA: MIT Press.

Monsell, S., \& Mizon, G. A. (2006). Can the task-cuing paradigm measure an endogenous task-set reconfiguration process? Journal of Experimental Psychology: Human Perception \& Performance, 32, 493-516.

Poljac, E., de HaAn, A., \& van Galen, G. P. (2006). Current task activation predicts general effects of advance preparation in task switching. Experimental Psychology, 53, 260-267.

SCHNEIDER, D. W., \& LOGAN, G. D. (2005). Modeling task switching without switching tasks: A short-term priming account of explicitly cued performance. Journal of Experimental Psychology: General, 134, 343-367.

\section{NOTE}

1. It may be worth noting that "success" was defined differently there than here. Logan and Bundesen (2003) evaluated their model fits descriptively with root mean squared deviations and correlations. Here, fits were tested against participant-level data using inferential statistics.

(Manuscript received December 4, 2005; revision accepted for publication April 17, 2006.) 